\title{
Respuesta del autor
}

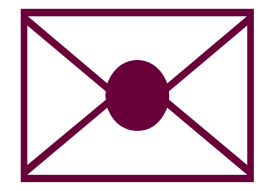

\section{CIRUGÍA DE EPILEPSIA EN EL HNGAI}

Respuesta del autor:

Agradezco el interés del doctor Rafael al comentar el artículo publicado por nosotros sobre el manejo quirúrgico en siete pacientes con epilepsia refractaria (Acta Med Per. 2014:31(4):199-212.

El planteamiento de trasplante de epiplón en el tratamiento de la epilepsia refractaria ha sido publicado en estudios de reporte de casos por Rafael y colaboradores. ${ }^{1}$

La teoría de la isquemia como factor etiopatogénico en la epilepsia es solo uno de los factores causales, existen en niños con mucha frecuencia las causas genéticas como la esclerosis tuberosa, displasias corticales que son alteraciones del desarrollo, los tumores de bajo grado de malignidad, las esclerosis secundarias a infecciones. ${ }^{2}$

La argumentación teórica planteada sobre la posible neovascularización y posible mejora de la función neuronal en el tejido epileptogénico resulta interesante y queda pendiente de demostración experimental en animales para evaluar con estudios histopatológicos pre y postrasplante de epiplón el cambio de aspecto y función.

La medicina basada en evidencias es la utilización consciente, explícita y juiciosa de la mejor evidencia clínica disponible para tomar decisiones sobre el cuidado de los pacientes individuales. Se viene utilizando en forma global desde su planteamiento, en 1992, por el Grupo de Trabajo de la Universidad MacMaster, en Canadá. ${ }^{3}$

El nivel de evidencia ha sido catalogado por varias instituciones, una de las más usadas a nivel mundial es la de la Universidad de Oxford: Centre for Evidence Based Medicine (CEBM), que clasifica los estudios según la siguiente tabla:

Con base en los estudios disponibles para cada tipo de terapia, se plantean las siguientes recomendaciones: Teniendo como referencia los estudios existentes a la fecha, la cirugía de epilepsia del tipo resectivo, luego de la identificación del foco epileptógeno a nivel temporal, tiene un nivel de recomendación A, según la Academia Americana de Neurología y la Sociedad Americana de Epilepsia. ${ }^{4}$

Las guías clínicas basadas en evidencia del Hospital Real de Melbourne de 2004, clasifican la cirugía de epilepsia del lóbulo temporal como nivel de evidencia grado ${ }^{5}$ al lograr en $63 \%$ de los pacientes libres de crisis a los 12 meses en comparación a $8 \%$ con fármacos antiepilépticos.

Si aplicamos los criterios del CEBM para clasificar los estudios publicados a la fecha sobre el trasplante de epiplón como tratamiento en epilepsia sería catalogado como nivel de evidencia 4, al existir solo reporte de serie de casos sin grupo comparativo. Por lo que quedaría pendiente realizar estudios diseñados con grupo control, aleatorios y prospectivos.

\begin{tabular}{|c|c|}
\hline Nivel & Tipo de estudio \\
\hline$\Delta 1 a$ & $\begin{array}{l}\text { Revisión sistemática de ensayos clínicos aleatorizados, } \\
\text { con homogeneidad }\end{array}$ \\
\hline$\triangle 2 b$ & $\begin{array}{l}\text { Ensayo clínico aleatorizado con intervalo de } \\
\text { confianza estrecho. }\end{array}$ \\
\hline$\Delta 1 c$ & Práctica clínica ("todos o ninguno")* \\
\hline$\Delta 2^{a}$ & $\begin{array}{l}\text { Revisión sistemática de estudios de cohortes, } \\
\text { con homogeneidad. }\end{array}$ \\
\hline$\Delta 2 b$ & $\begin{array}{l}\text { Estudio de cohortes o ensayo clínico } \\
\text { aleatorizado de baja calidad }{ }^{\star \star}\end{array}$ \\
\hline$\Delta 2 c$ & Outcomes research, ${ }^{\star \star \star}$ estudios ecológicos. \\
\hline$\triangle 3 a$ & $\begin{array}{l}\text { Revisión sistemática de estudios de casos y } \\
\text { controles, con homogeneidad. }\end{array}$ \\
\hline$\Delta 3 b$ & Estudio de casos y controles. \\
\hline$\triangle 4$ & $\begin{array}{l}\text { Serie de casos o estudios de cohortes y de } \\
\text { casos y controles de baja calidad }{ }^{\star \star \star \star}\end{array}$ \\
\hline$\triangle 5$ & $\begin{array}{l}\text { Opinión de expertos sin valoración crítica explícita, } 0 \\
\text { basados en la fisiología, bench research o first principles }\end{array}$ \\
\hline
\end{tabular}

\section{REFERENCIAS}

I. Rafael H, Mego R, Moromizato P, Garcia W. Omental transplantation for temporal lobe epilepsy: report of two cases. Neurol India. 2002;50(I):71-4.

2. Ko D. Epilepsy and seizures. Emedicine. 24 septiembre de 2014. emedicine.medscape. com/article/ I 84846-overview

3. Primo J. Niveles de evidencia y grados de recomendación. Enfermedad inflamatoria Intestinal al Día. 2003;2(2):39-42.

4. AAN. AAN Guideline Summary for clinicians. Treatments for Refractory Epilepsy. www. aan.com/professionals/practice/index.cfm.

5. Department of Neurology and Clinical Epidemiology and Health Service. Evaluation Unit. Royal Melbourne Hospital. Management of Epilepsy in Adults. Evidence Based Guidelines recommended for use in the Royal Melbourne Hospital. Review date: July 2004

\section{Marco Antonio Mejía-Tupa}

\begin{tabular}{|c|c|}
\hline Nivel & Tipo de estudio \\
\hline$\triangle A$ & Estudios de nivel 1 \\
\hline$\triangle B$ & $\begin{array}{l}\text { Estudios de nivel } 2-3 \text {, } \\
\text { o extrapolación de estudios de nivel } 1\end{array}$ \\
\hline$\triangle C$ & $\begin{array}{l}\text { Estudios nivel } 4 \text {, } \\
\text { o extrapolación de estudios de nivel 2-3 }\end{array}$ \\
\hline$\triangle D$ & $\begin{array}{l}\text { Estudios de nivel } 5 \text {, } \\
\text { o estudios no concluyentes de cualquier nivel }\end{array}$ \\
\hline
\end{tabular}

